TITLE:

\title{
Semiquantal Valence-Bond Wave Packet Description of Chemical Bonding
}

$\operatorname{AUTHOR}(S)$ :

Ando, Koji

CITATION:

Ando, Koji. Semiquantal Valence-Bond Wave Packet Description of Chemical Bonding. Bulletin of the Chemical Society of Japan 2009, 82(8): 975-983

\section{ISSUE DATE:}

2009

URL:

http://hdl.handle.net/2433/203035

\section{RIGHT:}

(c) 2009 The Chemical Society of Japan; Publisher permitted posting the accepted manuscript on this repositry:; この論文は出版社版でありません。引用の際には出版社版 をご確認ご利用ください。; This is not the published version. Please cite only the published version. 


\title{
Semiquantal valence-bond wavepacket description of chemical bonding*
}

\author{
Koji Ando \\ Department of Chemistry, Graduate School of Science, Kyoto University, \\ Kyoto 606-8502, Japan
}

\begin{abstract}
A semiquantal wavepacket modeling of electrons in chemical bonding is presented. It is based on the valencebond (VB) theory with non-orthogonal floating and breathing spherical Gaussian orbitals, simplified to treat many electrons by decoupled electron pair approximations (DPA) and core pseudopotentials (CPP). The extended Hamiltonian formalism offers pictorial interpretation and analysis in the extended phase space of the wavepacket center and width coordinates. The numerical calculations are demonstrated on the ground state potential energy surfaces of $\mathrm{H}_{2}, \mathrm{LiH}$, and $\mathrm{BeH}_{2}$. For $\mathrm{LiH}$, the perfect-pairing VB (VB-PP) calculation with the minimal orbitals gives an accurate potential energy curve of comparable quality with a correlated ab initio calculation. The two-electron VB calculation with a CPP underestimates the binding energy but gives qualitatively correct potential energy curves. For $\mathrm{BeH}_{2}$, the VB-PP with CPP gives reasonably accurate potential energy surface along both the stretching and bending coordinates. A few versions of DPA are developed and assessed, aiming toward large scale dynamic simulations. A scaling ansatz is introduced and examined on the bonding potential energy surfaces. The efficacy of the theory for studying linear and nonlinear electronic polarizations is also illustrated via an analysis of potential energy surfaces in the extended phase space.
\end{abstract}

\section{INTRODUCTION}

A goal of theoretical and computational chemistry is to develop approaches to the solution of full molecular timedependent Schrödinger equations treating both electronic and nuclear degrees of freedom as dynamical variables. This is obviously too demanding for chemical problems involving both degrees of freedom in non-trivial manners. A range of approximations and models are therefore deployed. One end is represented by high-level ab initio quantum chemical calculations with large scale electron-correlation methods; ${ }^{1,2}$ the other is statistical mechanical simulations by molecular dynamics (MD), Monte Carlo, and integral-equation methods. 3,4 The former are yet too expensive for the direct dynamics simulations of large systems, while the latter often employ simplified potential functions ignoring electronic structure alterations.

The hybrid of these two ends, the so-called QM/MM strategy, has been a major arena in the last decade. ${ }^{5,6}$ Nonetheless, consistent treatment of the boundary between QM (quantum mechanics) and MM (molecular mechanics) regions has been the bottleneck both technically and conceptually. Another growing area is the simulation of real-time electron dynamics by time-dependent Hartree-Fock, time-dependent density functional, and other methods. ${ }^{7-10}$ These are naturally more expensive than their time-independent counterparts.

While qualifying the promise of these state-of-the-art computations, ${ }^{11}$ in this work we investigate a different approach. It is motivated by the recent development of semiquantal time-dependent Hartree (SQTDH) theory for chemical dynamics in condensed phase. ${ }^{12-14}$ The SQTDH theory describes the wavefunction as a Hartree product of the squeezed coherent state Gaussian wavepackets. The structure and dy-

*Published in Bull. Chem. Soc. Jpn. Vol. 82, No. 8, 975-983 (2009). DOI: $10.1246 / \mathrm{bcsj} .82 .975$

(C) 2009 The Chemical Society of Japan namics of the wavefunction can be studied in a pictorial manner on a potential surface in an extended phase space formed by the wavepacket center and width coordinates. This has been demonstrated for coupled system-bath models ${ }^{12}$ and hydrogen bonding structures. ${ }^{13}$

As the Hartree approximation implies however, manyfermion systems were out of the scope of the SQTDH theory. We therefore attempt in this work to extend it to account for the antisymmetry of electronic wavefunctions. To this end, we exploit and combine the ideas of the valence bond (VB) theory, non-orthogonal floating and breathing orbitals, decoupled electron pair approximation (DPA), and core pseudopotentials (CPP). It is thus designed to achieve semi-quantitative correctness and applicability to large scale dynamic simulations.

The time-independent electronic part of the present theory is partially related to the FSGO (floating spherical Gaussian orbital) method. ${ }^{15}$ The main difference is the restricted Hartree-Fock (RHF) nature of the latter, contrasted with the VB idea of the former. On the other hand, the dynamic part is related to the fermion wavepacket MD simulations in nuclear and plasma physics. ${ }^{16-20}$ The distinction is in the treatment of the antisymmetry of the wavefunction and the DPA based on the VB coupling, reflected in the form of the Pauli potential. The present work shares the motivation with the eFF (electron force field) model by $\mathrm{Su}$ and Goddard, ${ }^{21}$ although the formulation and the resultant potential function are different.

In this first report, the basic framework of the theory is described and numerical applications are examined on the ground state potential energy surfaces of small molecules such as $\mathrm{H}_{2}, \mathrm{LiH}$, and $\mathrm{BeH}_{2}$. Applications to larger systems and to dynamic processes will be presented in the forthcoming publications.

Section II describes the theory for one and two electron systems, a scaling ansatz, and the CPP and DPA for many electron systems. Section III presents numerical applications and discussions. The paper concludes in Sec. IV. 


\section{THEORY}

\section{A. One electron atoms and molecules}

\section{Basic framework}

For simplicity, we set $\hbar=1$ and assume that the coordinates are mass-scaled. The trial wave function ${ }^{22-24}$ is defined in the coherent state spherical Gaussian form,

$$
\phi(\boldsymbol{q}, t)=N \exp \left\{A(t)|\boldsymbol{q}-\boldsymbol{x}(t)|^{2}+i \boldsymbol{p}(t) \cdot(\boldsymbol{q}-\boldsymbol{x}(t))\right\},
$$

in which

$$
A(t)=\frac{-1+2 i \rho(t) \pi(t)}{4 \rho(t)^{2}}
$$

and $N=\left(2 \pi \rho(t)^{2}\right)^{-\frac{3}{4}}$ is the normalization factor. The timedependent parameters $\boldsymbol{x}(t)$ and $\rho(t)$ describe the center and width of the wavepacket; $\boldsymbol{p}(t)$ and $\pi(t)$ are seen below to represent the conjugate momenta of $\boldsymbol{x}(t)$ and $\rho(t)$.

The equations of motion for the parameters are derived from the time-dependent variational theory, ${ }^{25}$ $\delta\left(\int L d t\right) / \delta X=0$, where $X$ represents all the parameters, and

$$
L=\left\langle\phi(t)\left|i \frac{\partial}{\partial t}-\hat{H}\right| \phi(t)\right\rangle
$$

with $\hat{H}=-(1 / 2) \partial^{2} / \partial q^{2}+V(q)$. This yields

$$
\begin{aligned}
& \dot{\boldsymbol{x}}=\partial H_{\mathrm{ext}} / \partial \boldsymbol{p}, \quad \dot{\boldsymbol{p}}=-\partial H_{\text {ext }} / \partial \boldsymbol{x} \\
& \dot{\rho}=\partial H_{\text {ext }} / \partial \pi, \quad \dot{\pi}=-\partial H_{\text {ext }} / \partial \rho,
\end{aligned}
$$

in which $H_{\text {ext }}$ is given by

$$
H_{\mathrm{ext}}=\frac{\boldsymbol{p}^{2}}{2}+\frac{\pi^{2}}{2}+\frac{3}{8 \rho^{2}}+\langle V\rangle,
$$

where $\langle V\rangle$ is the expectation value of $V$ with respect to $|\phi\rangle$. The classical Hamiltonian form of Eq. (4) suggests consideration of the phase space $(\boldsymbol{x}, \rho, \boldsymbol{p}, \pi)$. The key quantity is therefore the potential

$$
V_{\text {ext }}=\frac{3 \hbar^{2}}{8 m \rho^{2}}+\langle V\rangle
$$

in the extended configuration space $(\boldsymbol{x}, \rho)$. Note that $\hbar$ and the mass $m$ are retrieved in the first term of Eq (6). This term tends to broaden the wavepacket, with a stronger tendency when the mass is the lighter, and vanishes in the classical limit $\hbar \rightarrow 0$. The optimal stationary state wavepacket in the ground state is obtained simply by minimizing $V_{\text {ext }}$.

In Ref 24, the problem of constructing an extended potential for coupled degrees of freedom has been pointed out. The Hartree ansatz was examined in Ref. 12 together with the canonicity condition (Ref. 26) for the extended Hamiltonian form. It was found to be essentitally equivalent to the QHD2 (quantized Hamilton dynamics) ${ }^{27}$ and QCD (quantized cumulant dynamics $)^{28}$ theories by assuming decoupling of the degrees of freedom. In this work, the use of the spherical Gaussian form evades this problem for the three Cartesian coordinates of a particle. It also obviates the issue concerning the rotational invariance.

\section{Hydrogen-like atoms}

The theory is well illustrated on the hydrogen-like atoms. Hereafter, we apply the atomic unit $\hbar=e=m_{e}=1$. The potential for the electron at position $\boldsymbol{q}$ is

$$
V_{\mathrm{H}}(\boldsymbol{q})=-Z /|\boldsymbol{q}|,
$$

where the nucleus with atomic number $Z$ is placed at the origin. In this work, we treat the nucleus as a classical point charge (the Born-Oppenheimer approximation). The wavepacket treatment of nuclei will be treated elsewhere.

The semiquantal extended potential is derived straightforwardly as

$$
V_{\mathrm{H}}^{\mathrm{ext}}(\boldsymbol{x}, \rho)=\frac{3}{8 \rho^{2}}-\sqrt{\frac{2}{\pi}} \frac{Z}{\rho} F_{0}\left(\frac{|\boldsymbol{x}|^{2}}{2 \rho^{2}}\right),
$$

in which $F_{0}$ is the Boys function ${ }^{29}$ of order 0 defined by

$$
F_{0}(t)=\int_{0}^{1} e^{-t y^{2}} d y=\sqrt{\frac{\pi}{4 t}} \operatorname{erf}(\sqrt{t})
$$

The minimum of $V_{\mathrm{H}}^{\text {ext }}$ is found at $\boldsymbol{x}=0$ from the symmetry. Therefore, $V_{\mathrm{H}}^{\text {ext }}$ is a quadratic function of $1 / \rho$ whose minimum is at

$$
\rho=\frac{3}{4 Z} \sqrt{\frac{2}{\pi}}\left(\equiv \rho_{0}\right)
$$

which is 0.940 bohr for $Z=1$. The minimum energy is

$$
V_{\mathrm{H}}^{\mathrm{ext}}\left(0, \rho_{0}\right)=-\frac{4 Z^{2}}{3 \pi}
$$

which is ca $85 \%$ of the exact 1 s energy $E_{\mathrm{H}}=-Z^{2} / 2$. The same result has been found in Ref 30 . Here we will extend the analysis in the following sections to the scaling ansatz and the calculation of polarizability. A note on the virial theorem is included in Appendix B.

\section{Static polarizability}

The polarizability is calculated from the energy change induced by an electric field $\boldsymbol{F}$, which adds a field-electron interaction $-\boldsymbol{F} \cdot \boldsymbol{q}$ to the Hamiltonian. The numerical calculation is straightforward, and the effect is pictured as a deformation of the extended potential $V_{\text {ext }}$, as will be demonstrated in Sec. III D. Fortunately, the analytical solution is available for the hydrogen-like atoms, which is described below. 
TABLE I: Expectation values of some powers of the electron-nucleus radial distance $r$ for the ground state hydrogen-like atoms. $a \equiv a_{0} / Z$ where $a_{0}$ is the Bohr radius and $Z$ is the atomic number. $\tilde{\rho} \equiv \rho / Z$ and $\rho_{0}$ is from Eq. (10).

\begin{tabular}{lccc}
\hline \hline & exact & semiquantal & using $\rho_{0}$ \\
\hline$\langle r\rangle$ & $\frac{3}{2} a$ & $\sqrt{\frac{8}{\pi}} \tilde{\rho}$ & $\frac{3}{2} a$ \\
$\left\langle r^{2}\right\rangle$ & $3 a^{2}$ & $3 \tilde{\rho}^{2}$ & $\frac{27 \pi}{32} a^{2}$ \\
$\left\langle r^{-1}\right\rangle$ & $a^{-1}$ & $\sqrt{\frac{2}{\pi}} \tilde{\rho}^{-1}$ & $\frac{8}{3 \pi} a^{-1}$ \\
$\left\langle r^{-2}\right\rangle$ & $2 a^{-2}$ & $\tilde{\rho}^{-2}$ & $\frac{32}{9 \pi} a^{-2}$ \\
\hline
\end{tabular}

Let us assume that a uniform field is applied along the $z$ axis. As the static polarizability is defined in the weak field limit, we expand the Boys function in Eq. (8) around $\boldsymbol{x}=0$,

$$
V_{\mathrm{H}}^{\mathrm{ext}}(z, \rho ; F) \simeq \frac{3}{8 \rho^{2}}-\sqrt{\frac{2}{\pi}} \frac{Z}{\rho}\left(1-\frac{z^{2}}{6 \rho^{2}}\right)-F z .
$$

Minimizing along $z$,

$$
V_{\mathrm{H}}^{\mathrm{ext}}\left(z_{0}, \rho ; F\right)=V_{\mathrm{H}}^{\mathrm{ext}}(0, \rho ; 0)-\frac{3}{2 Z} \sqrt{\frac{\pi}{2}} \rho^{3} F^{2},
$$

where $z_{0}=(3 / Z) \sqrt{\pi / 2} \rho^{3} F$. The polarizability $\alpha$ is thus

$$
\alpha=\frac{3}{Z} \sqrt{\frac{\pi}{2}} \rho_{0}^{3}=\frac{81 \pi^{2}}{256 Z^{4}} \sim 3.12 / Z^{4},
$$

which is ca $69 \%$ of the value $9 / 2$ from the perturbation theory $^{31}$ for the hydrogen atom.

\section{Scaling ansatz}

The underestimate of the energy for the hydrogen-like atoms in Eq. (11) stems from the use of the Gaussian function. This is related to the cusp condition satisfied by the exact $1 \mathrm{~s}$ function but not by the Gaussian function. ${ }^{32}$ Nonetheless, we may explore scaling properties when the theory is consistent within the variational subspace.

Table I compares the expectation values of some powers of the electron-nucleus radial distance $r$ from the exact and the semiquantal theories for the ground state of the hydrogen-like atoms. As seen in Eq. (11), the energy is scaled as

$$
E_{\mathrm{H}}^{\mathrm{SQ}}=\frac{8}{3 \pi} E_{\mathrm{H}}^{\mathrm{exact}}
$$

This suggests that we introduce a scaling correction factor

$$
\gamma_{E} \equiv \frac{3 \pi}{8}
$$

for energies computed from the semiquantal theory.
For the length scale, a possible factor is suggested from the ratio of $\langle r\rangle$,

$$
\gamma_{L}^{\prime}=\frac{\langle r\rangle_{\text {exact }}}{\langle r\rangle_{\mathrm{SQ}}}=1
$$

Another possibility would be to refer to $\left\langle r^{-1}\right\rangle$,

$$
\gamma_{L}^{\prime \prime}=\frac{\left\langle r^{-1}\right\rangle_{\mathrm{SQ}}}{\left\langle r^{-1}\right\rangle_{\text {exact }}}=\frac{8}{3 \pi}
$$

Since the arithmetic and geometric means of $\gamma_{L}^{\prime}$ and $\gamma_{L}^{\prime \prime}$ give similar numbers, 0.924 and 0.921 , we choose the latter for simplicity,

$$
\gamma_{L} \equiv \sqrt{\gamma_{L}^{\prime} \gamma_{L}^{\prime \prime}}=\sqrt{\frac{8}{3 \pi}}
$$

These scaling corrections will be carried over and examined on chemical bondings in Sec. III.

\section{B. Two electron atoms and molecules}

Here we summarize the Heitler-London ${ }^{33}$ (HL) VB framework. The main purpose is to fix the notations. The HL-VB wavefunction for two electrons is

$$
\begin{array}{r}
\psi_{a b}^{\mathrm{HL}}(1,2)=\frac{1}{\sqrt{2\left(1+S_{a b}^{2}\right)}}\left(\phi_{a}\left(\boldsymbol{q}_{1}\right) \phi_{b}\left(\boldsymbol{q}_{2}\right)+\phi_{b}\left(\boldsymbol{q}_{1}\right) \phi_{a}\left(\boldsymbol{q}_{2}\right)\right) \\
\times \frac{1}{\sqrt{2}}(\alpha(1) \beta(2)-\beta(2) \alpha(1)) . \quad(20)
\end{array}
$$

As we restrict the numerical calculations to the stationary wavefunctions in this work, the momentum parameters $\boldsymbol{p}$ and $\pi$ in Eq. (1) are nullified and the spatial orbitals $\phi_{a}(\boldsymbol{q})$ are specified by the wavepacket center $\boldsymbol{x}_{a}$ and width $\rho_{a} . \alpha$ and $\beta$ are the spin functions. $S_{a b}$ is the overlap integral between $\phi_{a}$ and $\phi_{b}$.

The corresponding electronic energy is given by

$E_{a b}^{\mathrm{HL}}=\frac{1}{1+S_{a b}^{2}}\left(h_{a a}+h_{b b}+2 S_{a b} h_{a b}+(a a \mid b b)+(a b \mid a b)\right)$,

where $h_{a b}$ is the one-electron integral consisting of the kinetic energy and the electron-nuclear potential. $(a a \mid b b)$ and $(a b \mid a b)$ are the two-electron Coulomb and exchange integrals. These integrals are summarized in Appendix A.

\section{Many-electron systems and DPA}

As is well-known, the complexity of the non-orthogonal VB calculation grows rapidly along with the number of electrons. It is thus essential to devise efficient approximations in order to realize large scale simulations. In this section, we develop and examine a few versions of DPA. To this end, it is useful to work on four electron systems for which the VB treatment is still handy (with $4 !=24$ matrix elements). 
In the first approximation, we show that the VB energy can be simplified to a pairwise form by rearranging the energy expression and introducing the Mulliken approximation ${ }^{34}$ to the integrals. This is further simplified to a form that contains terms resembling the so-called Pauli potential. ${ }^{16-18}$ In the third approximation, the wavefunction is completely decoupled to a product of the HL-VB pair functions.

\section{1. $V B$ energy for four electrons}

The energy of the perfect-pairing VB (VB-PP) wavefunction $^{35}$ in which orbital pairs $(a, b)$ and $(c, d)$ are coupled in the singlet configuration is given by ${ }^{36}$

$$
E_{a b \cdot c d}^{\mathrm{VBPP}}=\frac{1}{\Delta}\left(Q+J_{2}+J_{3}+J_{4}\right)
$$

where

$$
Q=\sum_{a} h_{a a}+\sum_{a<b}(a a \mid b b)
$$

and $J_{n}(n=2,3,4)$ represents $n$-electron exchange integrals. For example, $J_{2}$ is given by

$$
J_{2} \equiv J_{a b}+J_{c d}-\left(J_{a c}+J_{a d}+J_{b c}+J_{b d}\right) / 2,
$$

where

$$
\begin{gathered}
J_{a b} \equiv\langle a b c d|H| b a c d\rangle=2 h_{a b} S_{a b}+\left(h_{c c}+h_{d d}\right) S_{a b}^{2} \\
+(a b \mid b a)+2\{(a b \mid c c)+(a b \mid d d)\} S_{a b}+(c c \mid d d) S_{a b}^{2} .
\end{gathered}
$$

The normalization factor is given by

$$
\Delta=1+S_{a b}^{2}+S_{c d}^{2}-\left(S_{a c}^{2}+S_{a d}^{2}+S_{b c}^{2}+S_{b d}^{2}\right) / 2+S_{3}+S_{4} .
$$

The formulas for the third and fourth-order exchange and overlap integrals $J_{3}, J_{4}, S_{3}$, and $S_{4}$ are rather lengthy, for which we would refer to Ref. 36 .

The two-electron part of $Q$ is the Coulomb interaction between electron distributions $\left|\phi_{a}\right|^{2}$ and $\left|\phi_{b}\right|^{2}$. The calculation of the pairwise interactions of this sort is already the bottleneck in large scale simulations. In this sense, the three- and four-center integrals such as $(a b \mid c c)$ and $(a b \mid c d)$ in $J_{n}(n=$ $2,3,4)$ will impose additional and higher computational demands. We shall thus seek for approximations to reduce the energy to simpler forms.

\section{DPA-1: integral approximation to the VB energy}

In the first approximation, we neglect the higher order terms $J_{3}, J_{4}, S_{3}$, and $S_{4}$ and rewrite $J_{2}$ in Eq. (22) as

$$
\begin{aligned}
E_{a b \cdot c d}^{\mathrm{VBPP}} & \simeq \frac{1}{\Delta}\left(Q+J_{2}\right) \\
= & Q-\frac{1}{\Delta}\left[S_{a b}^{2} j_{a b}+S_{c d}^{2} j_{c d}\right. \\
& \left.-\left(S_{a c}^{2} j_{a c}+S_{a d}^{2} j_{a d}+S_{b c}^{2} j_{b c}+S_{b d}^{2} j_{b d}\right) / 2\right]
\end{aligned}
$$

where

$$
j_{a b} \equiv Q-J_{a b} / S_{a b}^{2}
$$

This still contains the three-center integrals in $J_{a b}$. Nonetheless, by applying the Mulliken approximation, ${ }^{34}$

$$
\begin{aligned}
(a b \mid c d) \simeq & \left(S_{a b} / 2\right)\left(S_{c d} / 2\right) \\
& \times[(a a \mid c c)+(a a \mid d d)+(b b \mid c c)+(b b \mid d d)]
\end{aligned}
$$

we find $j_{a b}$ reduces to a pairwise quantity of $\phi_{a}$ and $\phi_{b}$,

$$
j_{a b} \simeq h_{a a}+h_{b b}-2 h_{a b} / S_{a b}+(a a \mid b b)-(a b \mid b a) / S_{a b}^{2}
$$

The use of Eqs. (27) and (30) will be called DPA-1.

\section{Pauli potential form (DPA-2)}

We may further approximate the denominator $\Delta$ in Eq. (27) to decouple into

$$
\begin{aligned}
& E_{a b \cdot c d}^{\mathrm{DPA} 2}=Q-\frac{S_{a b}^{2}}{1+S_{a b}^{2}} j_{a b}-\frac{S_{c d}^{2}}{1+S_{c d}^{2}} j_{c d}+\frac{S_{a c}^{2} / 2}{1-S_{a c}^{2} / 2} j_{a c} \\
& +\frac{S_{a d}^{2} / 2}{1-S_{a d}^{2} / 2} j_{a d}+\frac{S_{b c}^{2} / 2}{1-S_{b c}^{2} / 2} j_{b c}+\frac{S_{b d}^{2} / 2}{1-S_{b d}^{2} / 2} j_{b d}
\end{aligned}
$$

which will be called DPA-2. The last four terms have a form similar to the Pauli potential, ${ }^{16-18}$ but with the extra factor $1 / 2$ to the overlap integrals. This factor reflects the spin couplings between the electrons in different pairs; the spins in orbitals $\phi_{a}$ and $\phi_{c}$ may be either parallel or anti-parallel, e.g., $\alpha \beta \cdot \alpha \beta$ or $\alpha \beta \cdot \beta \alpha$, in $a b \cdot c d$. In contrast, the factor $1 / 2$ is absent in the previous Pauli potentials, as they are derived from the triplet (parallel) spin coupling.

\section{DPA-3: decoupled product wavefunction}

In the third approximation, we decouple the wavefunction into the product form

$$
\psi(1,2,3,4) \simeq \psi_{a b}^{\mathrm{HL}}(1,2) \psi_{c d}^{\mathrm{HL}}(3,4) .
$$

The energy of this wavefunction consists of the HL-VB energies of the $a b$ and $c d$ pairs of the form Eq. (21) and the interaction between them. The latter contains the three- and four-center integrals. Introducing the Mulliken approximation and $j_{a b}$ of Eq. (30), however, the energy is again reduced to a pairwise form,

$$
E_{a b \cdot c d}^{\mathrm{DPA} 3}=Q-\frac{S_{a b}^{2}}{1+S_{a b}^{2}} j_{a b}-\frac{S_{c d}^{2}}{1+S_{c d}^{2}} j_{c d},
$$

which will be called DPA-3. This coincides with the first three terms in the right hand side of Eq. (31), although the derivations are different as we have seen. The energy formulas appear to suggest that DPA-2 is an intermediate between DPA-1 and DPA-3. This will be examined numerically in Sec. III. 


\section{Pseudopotentials for core electrons}

We will see in Sec. III that the VB-PP energy of Eq. (22) gives a good description of the core-valence interaction. Nonetheless, CPPs will be useful for large scale simulations.

In this work, we employ a CPP of the form

$$
V_{\mathrm{cpp}}(r)=-\frac{Z-Z_{c}}{r}\left(1-A_{c} \exp \left(-r^{2} / 4 \rho_{c}^{2}\right)\right),
$$

where $Z_{c}$ is the number of core electrons. The parameter $\rho_{c}$ represents the width of the potential. This potential is Coulombic in the large $r$ region, but its behavior at $r=0$ is singular at $A_{c}=1 ; V_{\mathrm{cpp}}(0)$ vanishes for $A_{c}=1$, but diverges to $\pm \infty$ for $A_{c}>1$ and $A_{c}<1$.

In the calculations in Sec. III, we only use $A_{c}=1$ for simplicity. This choice follows the previous studies ${ }^{37-39}-$ although their functional forms are designed for use with the plane wave basis - that anticipate cancellation of kinetic and potential energies in the core region. In this regard, Eq. (34) with $A_{c}=1$ is distinguished from the ECP (effective core potential) popular in quantum chemistry.

With $A_{c}=1$, we need one parameter $\rho_{c}$ per element. Nonetheless, considering the dependence $\rho_{0} \propto Z^{-1}$ in Eq. (10), we may seek a common parameter $\tilde{\rho}_{c}$ to scale as $\rho_{c}=$ $\tilde{\rho}_{c} / Z$ for elements in a row in the periodic table.

\section{NUMERICAL RESULTS AND DISCUSSION}

In this work, the energy minimization was carried out by the Brent's method ${ }^{40}$ that does not require derivatives.

\section{A. Hydrogen molecule}

The importance of orbital breathing in the covalent bonding of $\mathrm{H}_{2}$ has been known for a long time. ${ }^{41}$ The effect of orbital floating was studied with both the VB and the MOCI (molecular orbital-configuration interaction) methods, and was concluded to be minor. ${ }^{30,42,43}$ Then, presumably due to the historical prevalence of the MO-CI methods, the orbital floating seems to have been paid less attention in the VB compared to the MO-CI. ${ }^{44-48}$ Here, we briefly revisit the problem within the present framework, aiming to set up a groundwork for the later sections.

Figure 1 (a) shows the potential energy curves of $\mathrm{H}_{2}$ from various calculations. It is seen that the energy and length scalings discussed in Sec. II A shift the curve closer to the accurate calculation by Kolos and Wolniewicz. ${ }^{49}$ The equilibrium bond length is $1.557 \mathrm{bohr}$ and $1.435 \mathrm{bohr}$ before and after the scaling, compared to 1.4011 bohr by Kolos-Wolniewicz.

For comparison, the RHF and unrestricted Hartree-Fock (UHF) calculations are included. The RHF scheme corresponds to the FSGO method. ${ }^{15}$ We also examined the mixing of an ionic VB function

$$
\frac{1}{\sqrt{2\left(1+S_{a b}^{2}\right)}}\left(\phi_{a} \phi_{a}+\phi_{b} \phi_{b}\right)(\alpha \beta-\beta \alpha) / \sqrt{2} \text {. }
$$
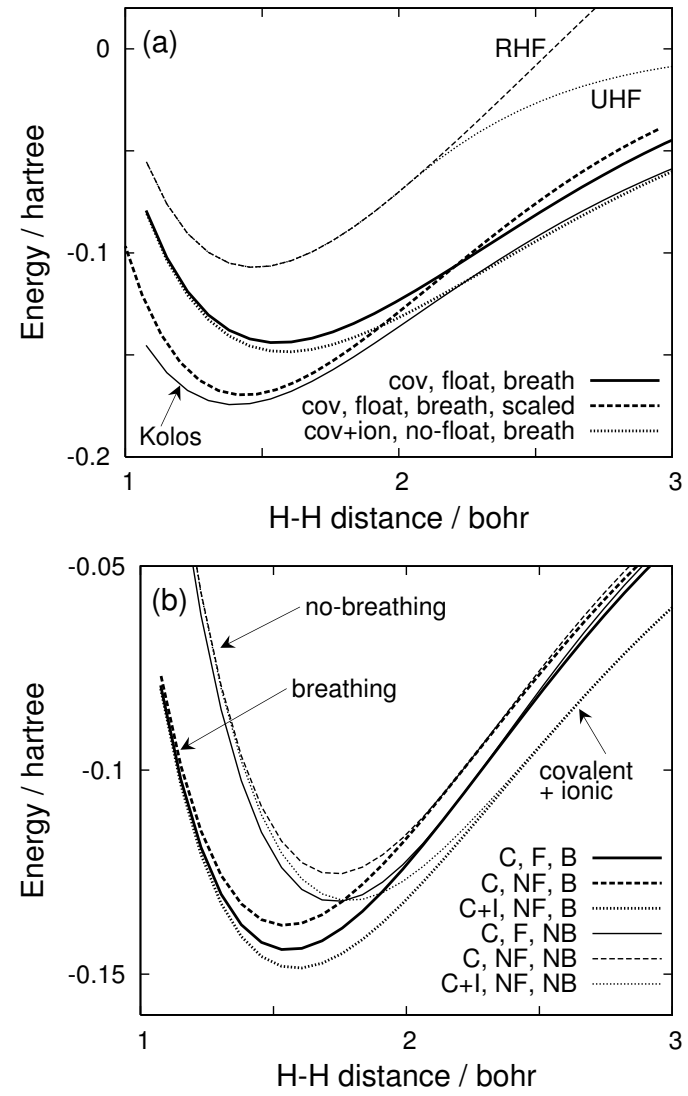

FIG. 1: Potential energy curves of hydrogen molecule. (a) Thick solid: covalent VB with floating and breathing orbitals (SQ-HLVB). Thick dashed: SQ-HLVB with the energy and length scalings. Thick dotted: covalent + ionic VB, no-floating but breathing. Thin solid: Kolos-Wolniewicz. Thin dashed: RHF, floating and breathing. Thin dotted: UHF, floating and breathing. (b) Thick solid: covalent only (C), floating (F) and breathing (B). Thick dashed: covalent only, no-floating (NF) but breathing. Thick dotted: covalent + ionic $(\mathrm{C}+\mathrm{I})$, no-floating but breathing. Thin solid: covalent only, floating but no-breathing (NB). Thin dashed: covalent only, no-floating and no-breathing. Thin dotted: covalent + ionic, no-floating and nobreathing.

In this case, the wavepacket centers are fixed at the nuclear positions, which would be in accord with the idea of the 'ionic' function. The mixing coefficients of the covalent and ionic components are fully optimized. This is thus equivalent to the full $\mathrm{CI}$ in the minimal breathing basis. As seen in the figure, the mixing of the ionic component lowers the energy particularly in the region of longer internuclear distance.

The effects of orbital floating and breathing and of the ionic function are compared in Fig. 1 (b). It is seen that both the orbital floating and the ionic component lower the energy but do not change much the equilibrium bond length. In contrast, the orbital breathing notably lowers the energy and shortens the equilibrium bond length. 


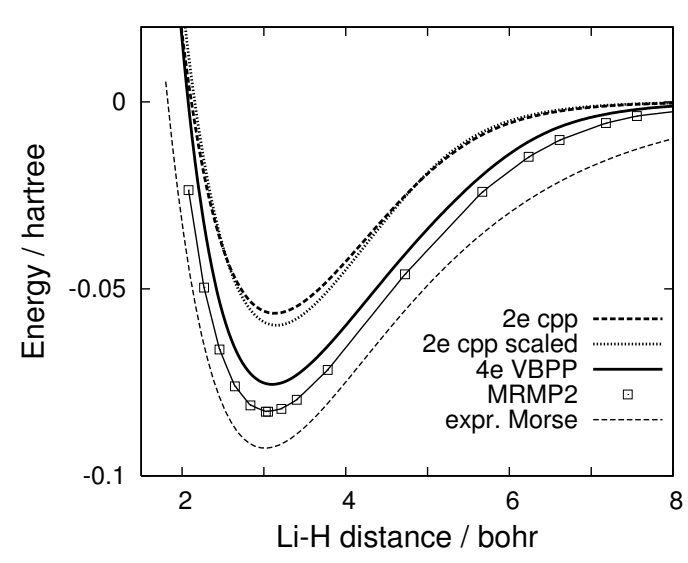

FIG. 2: Potential energy curves of $\mathrm{LiH}$. Thick solid: Four electron SQ-VBPP. Thick dashed: Two electron SQ-HLVB with CPP of $\rho_{c}=$ 0.7 bohr. Thick dotted: Two electron scaled SQ-HLVB with CPP of $\rho_{c}=0.8$ bohr. Box marks: MRMP2/cc-pVDZ. Thin dotted: Morse function from experimental spectroscopic constants.

\section{B. LiH: Assessment of VB-PP and CPP}

Figure 2 shows the potential energy curves of $\mathrm{LiH}$ from the four-electron VB-PP and the HL-VB with use of the CPP. For comparison, the Morse function derived from the experimental spectroscopic constants ${ }^{50,51}$ and the MRMP2 (multi-reference second-order Møller-Plesset perturbation) calculation $^{52}$ with the cc-pVDZ basis set are included. We used the program GAMESS ${ }^{53}$ for the MRMP2 calculation. In the CASSCF (complete active space self-consistent field) calculation prior to the MRMP2, the 1s core orbital of Li was frozen and two electrons were distributed in the six valence orbitals.

As seen in the figure, the VB-PP calculation gives the result close and parallel to the MRMP2 and the experimental Morse potentials. This is remarkable for the compactness of the wavefunction formed by one floating and breathing orbital per electron. The result also indicates that the present VB-PP wavefunction is free from the problem in describing the corevalence interaction pointed out in the FSGO method. ${ }^{54,55}$

To the VB-PP calculation shown in the figure, we did not apply the scaling of Sec. II A, but the equilibrium bond length is already close to the accurate references. This seems to imply that the screening by the 1 s core electrons is effective such that the error due to the lack of wavefunction cusp is masked for the valence electron, in contrast with the case of $\mathrm{H}_{2}$. (See also Fig. 3 discussed below.)

In the calculation with CPP, we searched the optimal value of the parameter $\rho_{c}$ with an increment of 0.1 bohr and found that $\rho_{c}=0.7$ bohr gives the thick dashed curve in the figure. In general, the smaller $\rho_{c}$ yields the shorter equilibrium bond length. Therefore, we also examined slightly larger $\rho_{c}=0.8$ bohr combined with the scaling ansatz discussed in Sec. II A, as displayed by the thick dotted curve. As seen, these two calculations give similar results that are both reasonably parallel to the MRMP2 and the experimental Morse potentials. The
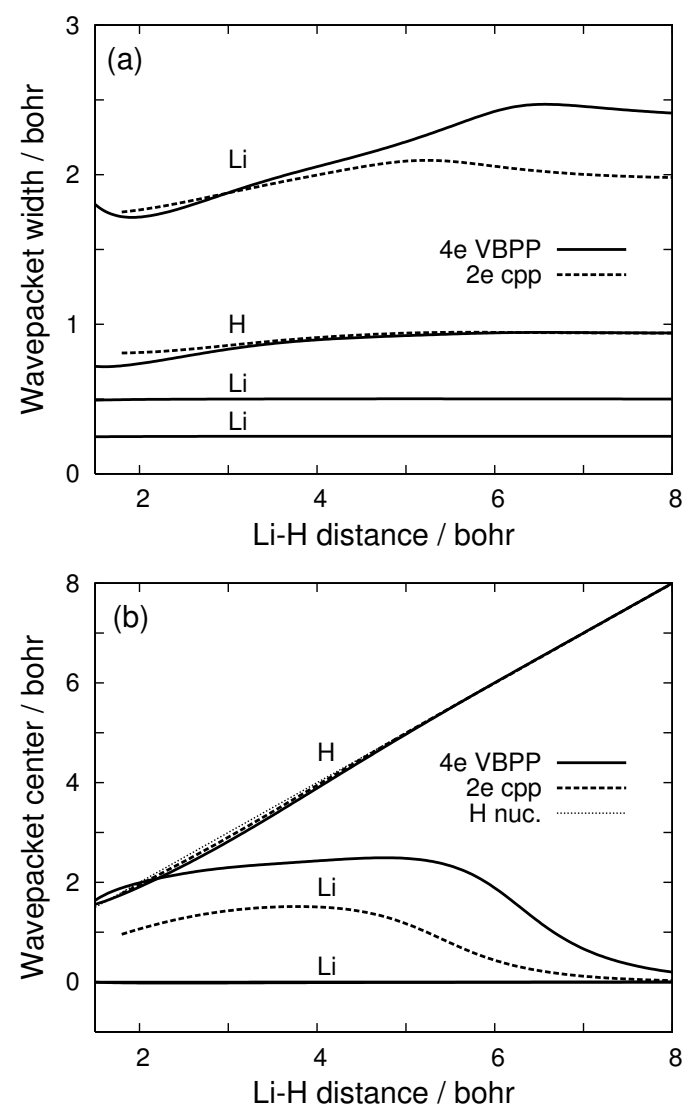

FIG. 3: Wavepacket parameters for LiH. (a) Wavepacket widths. (b) Wavepacket centers measured from the Li nucleus. Solid: Four electron SQ-VBPP. Dashed: Two electron SQ-HLVB with CPP of $\rho_{c}=0.7$ bohr. In (b), the dotted line denotes the position of the proton.

underestimate of the binding energy is seemingly due to the use of CPP.

Figure 3 displays the wavepacket widths (a) and the center positions (b). The latter are measured from the Li nucleus. The nearly constant lines in (a) at $\rho=0.25$ and 0.50 bohr represent the Li 1s core electrons in the VB-PP calculation. These are centered at the Li nucleus as seen in (b). The remaining two orbitals represent the valence electrons assigned to $\mathrm{Li}$ and $\mathrm{H}$. Both shrink as the atoms approach to each other, indicating the increase of the kinetic energy associated with the bond formation. This is in accord with the virial theorem (see also $\mathrm{Sec}$. III D). In the region of Li-H distance longer than 5 bohr, the solid and dashed curves for the width of Li valence orbital start to deviate notably. In the same region, the orbital center starts to move from the internuclear region toward the $\mathrm{Li}$ nucleus. The deviation thus comes from the different descriptions of the core-valence interaction. For the $\mathrm{H}$ orbital, the two calculations give similar width in the entire bond length region, and the orbital center stays close to the proton nucleus. 

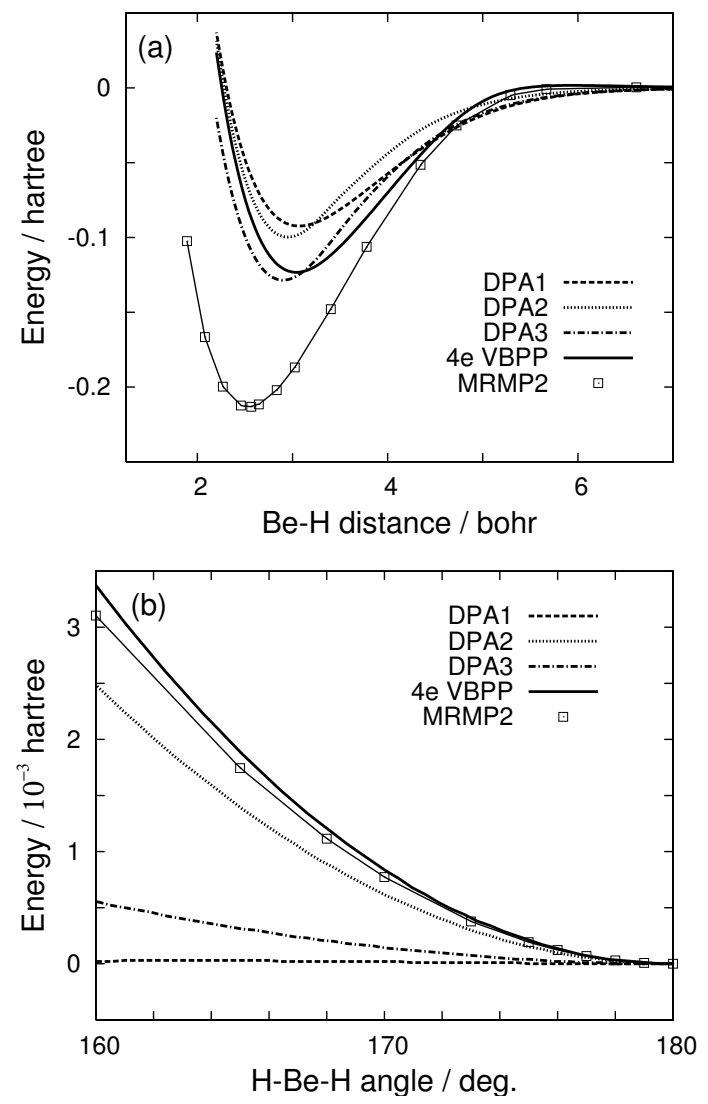

FIG. 4: Potential energy curves of $\mathrm{BeH}_{2}$. (a) Along the symmetric stretch of the $\mathrm{BeH}$ distance. (b) Along the $\mathrm{H}-\mathrm{Be}-\mathrm{H}$ angle at $r(\mathrm{BeH})=2.566$ bohr. Solid: Four electron SQ-VBPP. Dashed: DPA1. Dotted: DPA-2. Dash-dotted: DPA-3. Box marks: MRMP2/ccpVDZ.

\section{C. $\mathrm{BeH}_{2}$ : Assessment of DPAs}

Figure 4 shows the potential energy curves of $\mathrm{BeH}_{2}$ along the Be-H symmetric stretch distance and the $\mathrm{H}-\mathrm{Be}-\mathrm{H}$ bending angle. The three versions of DPA and VB-PP are compared against MRMP2. A CPP with $\rho_{c}=0.6 \mathrm{bohr}$ is used for the $1 \mathrm{~s}$ core of Be. This $\rho_{c}$ is related via $\rho_{c}=\tilde{\rho}_{c} / Z$ to the $\rho_{c}=0.8$ bohr for $\mathrm{Li}$ in the previous section.

As noted before, the underestimate of the binding energy seems to come from the use of CPP. Similarly to the cases of $\mathrm{H}_{2}$ and $\mathrm{LiH}$, the scaling prescription improves the results, but the curves shown in the figure are the unscaled ones.

In Sec. II C, we have noted that the energy formula for DPA-2 appears to be the intermediate between DPA-1 and DPA-3. This simple view is, however, not supported by the results in Fig. 4. The shapes of the potential look similar between VB-PP and DPA-1 and between DPA-2 and DPA-3.

Regarding the bending potential, VB-PP is closest to the MRMP2 reference, and DPA-2 comes next. The success of DPA-2 is presumably due to some cancellation of errors. To clarify this, we have checked the wavepacket center and width
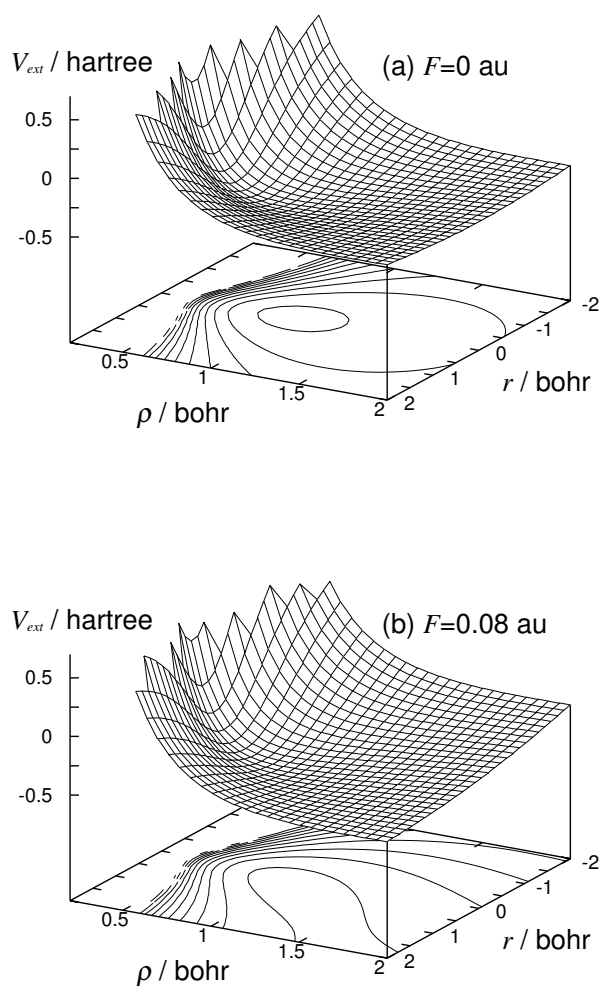

FIG. 5: Extended potential $V_{\text {ext }}(r, \rho)$ for hydrogen atom. (a) isolated atom. (b) under electric field $F=0.08$ a.u.

parameters, but could not draw a definite conclusion. Further investigation is needed on this issue, along with the extension to larger systems.

\section{Extended potential surface}

Figure 5 shows the deformation of the extended potential energy surface $V_{\mathrm{H}}^{\text {ext }}(r, \rho)$ (Eq. (8) with $r=|\boldsymbol{x}|$ ) of a hydrogen atom under a uniform static electric field. In Fig. 5 (b) with $F=0.08$ a.u., the minimum energy point is located at $r=0.293$ bohr and $\rho=0.983$ bohr, the latter being larger than the $\rho_{0}=0.940$ bohr of Eq. (10). The energy change from the isolated atom is computed to be -0.01077 hartree, which is larger in magnitude than that calculated from the polarizability of Eq. (14), $-\alpha F^{2} / 2=-0.00999$ hartree. This reflects the nonlinearity of the polarization under this strong field.

The extended potential energy surfaces for the electrons in $\mathrm{H}_{2}$ are displayed in Fig. 6. The position of the electron wavepacket center $x$ is measured from the center of the two nuclei and along the H-H bond. Figures 6 (a) and 6 (b) are calculated at the $\mathrm{H}-\mathrm{H}$ distance $R=1.4$ and $2.5 \mathrm{bohr}$, compared to the equilibrium bond length $R=1.557$ bohr obtained 

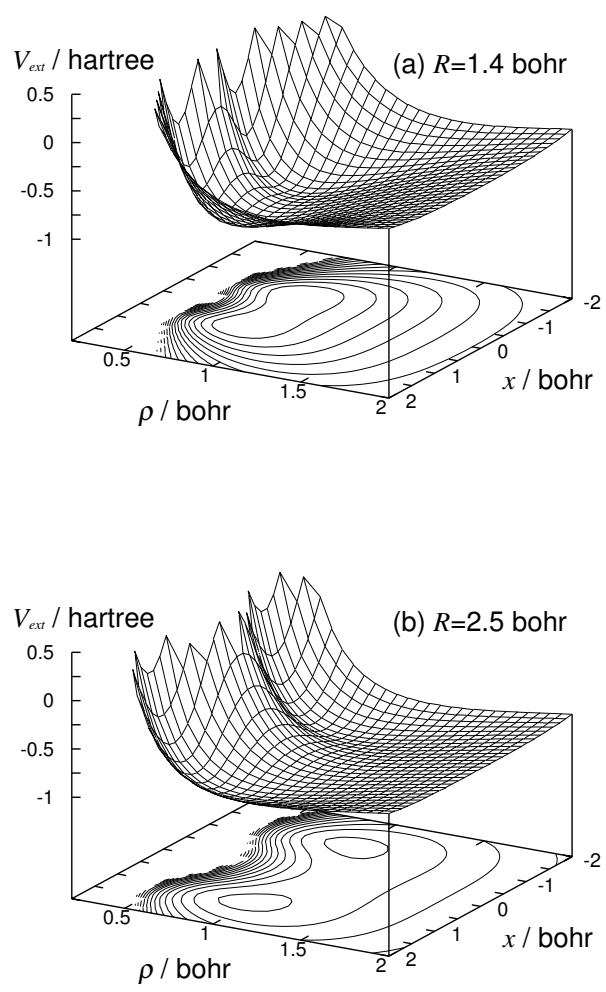

FIG. 6: Extended potential $V_{\text {ext }}(x, \rho)$ for hydrogen molecule. (a) The $\mathrm{H}-\mathrm{H}$ distance $R=1.4$ bohr. (b) $R=2.5$ bohr.

TABLE II: Results from the SQ-HLVB calculation on hydrogen molecule. $\Delta x \equiv R / 2-x$ is the shift of the wavepacket center from the nuclei. Lengths in bohr, energy in hartree.

\begin{tabular}{lcccccc}
\hline \hline$R$ & $x$ & $\Delta x$ & $\rho$ & $E$ & $-\langle V\rangle /\langle T\rangle$ & $\langle V\rangle / 2 E$ \\
\hline 1.4 & 0.600 & 0.100 & 0.805 & -0.9882 & 1.91569 & 1.04603 \\
1.557 & 0.668 & 0.111 & 0.837 & -0.9929 & 2.00002 & 0.99999 \\
2.5 & 1.135 & 0.116 & 0.971 & -0.9303 & 2.28857 & 0.88803 \\
\hline \hline
\end{tabular}

in Sec. III A.

The corresponding parameters and energies are listed in Table II, together with the quantities pertaining to the virial theorem. As the $\mathrm{H}-\mathrm{H}$ bond is stretched, the wavepacket centers of the electrons move closer to the nuclear position, and the wavepacket widths become larger. The virial theorem is satisfied at the equilibrium bond length, as expected for the fully optimized floating and breathing orbitals. The change of the virial ratio $-\langle V\rangle /\langle T\rangle$ implies that the raise of the energy in the shorter bond length is due to the increase of the kinetic energy, which is connected to the behavior of the wavepacket width $\rho$. (See also the discussion around Fig. 3.)

For general many-electron systems, the adiabatic elimina- tion of uninteresting degrees of freedom will be useful, similarly to that demonstrated for the system-bath models. ${ }^{12}$ The dynamic polarization can be studied by the Hamiltonian trajectory on the extended potential surface within the present framework. These extensive subjects will be treated in separate publications.

\section{CONCLUDING REMARKS}

A study on the semiquantal wavepacket modeling of electrons in chemical bonding has been presented. In contrast with the traditional MO picture based on the delocalized single-particle mean-field approximation, the VB framework fits well with the localized electron wavepacket ansatz. The floating and breathing spherical Gaussian orbitals are found to give the potential energy surfaces of reasonable accuracy with the minimal number of orbitals. Emerging is thus not simply the localized orbital picture but the corpuscular picture of the electrons.

On developing the DPA and CPP, our motivation was promoted by the empirical success of the VSEPR (valence shell electron pair repulsion) theory. ${ }^{56}$ Although the VB-PP result on $\mathrm{LiH}$ is encouraging, the examinations of the DPAs and CPP indicates that further analysis and development are needed for extension to larger systems. Since the orbitals are inherently localized in the present framework, we may, for example, include the higher order terms in the VB energy only for locally adjacent pairs.

Other directions in which to proceed will be toward dynamic simulations and the semiquantal treatment of protons and other light nuclei. The latter is related to the nuclearorbital methods, ${ }^{57-59}$ which are more elaborate and hence intriguing to compare. Work on these extensions will be reported in forthcoming publications.

\section{Acknowledgments}

This work has been supported by KAKENHI in Priority Areas "Molecular Theory for Real Systems" (No. 190296) and "Emergence of Highly Elaborated $\pi$-space and its Function" (No. 20106017).

\section{Appendix A: Integral formulas}

Here we compile the formulas for the energy integrals. The one-electron integrals consist of the kinetic and electronnuclear terms,

$$
h_{a b}=T_{a b}+V_{a b}^{\mathrm{ne}},
$$

where

$$
T_{a b}=\frac{1}{4\left(\rho_{a}^{2}+\rho_{b}^{2}\right)}\left(3-\frac{\left|\boldsymbol{x}_{a}-\boldsymbol{x}_{b}\right|^{2}}{2\left(\rho_{a}^{2}+\rho_{b}^{2}\right)}\right) S_{a b}
$$


with the overlap integral

$$
S_{a b}=\left(\frac{2 \rho_{a} \rho_{b}}{\rho_{a}^{2}+\rho_{b}^{2}}\right)^{\frac{3}{2}} \exp \left(-\frac{\left|\boldsymbol{x}_{a}-\boldsymbol{x}_{b}\right|^{2}}{4\left(\rho_{a}^{2}+\rho_{b}^{2}\right)}\right)
$$

and

$$
\begin{aligned}
& V_{a b}^{\mathrm{ne}}=\left\langle\phi_{a}(\boldsymbol{q})\left|\sum_{I}-\frac{Z_{I}}{\left|\boldsymbol{q}-\boldsymbol{R}_{I}\right|}\right| \phi_{b}(\boldsymbol{q})\right\rangle= \\
& -\left(\frac{\rho_{a}^{2}+\rho_{b}^{2}}{\pi \rho_{a}^{2} \rho_{b}^{2}}\right)^{\frac{1}{2}} S_{a b} \sum_{I} Z_{I} F_{0}\left(\frac{\rho_{a}^{2}+\rho_{b}^{2}}{4 \rho_{a}^{2} \rho_{b}^{2}}\left|\boldsymbol{x}_{p}-\boldsymbol{R}_{I}\right|^{2}\right),
\end{aligned}
$$

in which $\boldsymbol{R}_{I}$ and $Z_{I}$ denote the nuclear coordinates and the atomic numbers. $\boldsymbol{x}_{p}$ is the center of the product $\phi_{a} \phi_{b}$ defined by

$$
\boldsymbol{x}_{p}=\frac{\rho_{b}^{2} \boldsymbol{x}_{a}+\rho_{a}^{2} \boldsymbol{x}_{b}}{\rho_{a}^{2}+\rho_{b}^{2}} .
$$

The two-electron Coulomb and exchange integrals are calculated from

$$
(a a \mid b b)=\left(\frac{2}{\pi\left(\rho_{a}^{2}+\rho_{b}^{2}\right)}\right)^{\frac{1}{2}} F_{0}\left(\frac{\left|\boldsymbol{x}_{a}-\boldsymbol{x}_{b}\right|^{2}}{2\left(\rho_{a}^{2}+\rho_{b}^{2}\right)}\right)
$$

and

$$
(a b \mid a b)=\left(\frac{\rho_{a}^{2}+\rho_{b}^{2}}{2 \pi \rho_{a}^{2} \rho_{b}^{2}}\right) S_{a b}^{2} .
$$

The above formulas will be sufficient to fix the notations and to compare with the previous fermion MD methods. For the three- and four-center two-electron integrals, use of the general formula ${ }^{60}$ and the conversion between the Gaussian exponents and the width parameters $\rho$ is convenient.

\section{Appendix B: Virial theorem for hydrogen-like atoms}

For stationary wavefunctions, we may regard the first term of Eq. (8) as the kinetic energy part, which is denoted by $T$. With the variationally optimized $\boldsymbol{x}=0$ and $\rho=\rho_{0}$ of Eq. (10) for the hydrogen-like atoms in Sec. II A, we find

$$
\langle T\rangle=\frac{3}{8 \rho_{0}^{2}}=\frac{4 Z^{2}}{3 \pi}
$$

and

$$
\langle V\rangle=-\sqrt{\frac{2}{\pi}} \frac{Z}{\rho_{0}}=-\frac{8 Z^{2}}{3 \pi} .
$$

Therefore, the virial theorem for Coulomb systems

$$
2\langle T\rangle+\langle V\rangle=0
$$

is satisfied. The virial theorem for $\mathrm{H}_{2}$ is examined in Sec. III D.
${ }^{1}$ K. Hirao, ed., "Recent Advances in Multireference Methods," World Scientific, Singapore (1999).

${ }^{2}$ T. Helgaker, P. Jørgensen, and J. Olsen, "Molecular ElectronicStructure Theory," Wiley, Chichester (2000).

${ }^{3}$ M. P. Allen and D. J. Tidesley, "Computer Simulation of Liquids," Clarendon, Oxford (1987).

${ }^{4}$ F. Hirata, ed., "Molecular Theory of Solvation," Kluwer Academic, Dordrecht (2003).

${ }^{5}$ K. Yasuda and D. Yamaki, J. Chem. Phys., 121, 3964 (2004).

${ }^{6}$ H. Lin and D. G. Truhlar, Theor. Chem. Acc., 117, 185 (2007).

7 H. B. Schlegel, S. M. Smith, and X. Li, J. Chem. Phys., 126, 244110 (2007)

${ }^{8}$ K. Burke, J. Werschnik, and E. K. U. Gross, J. Chem. Phys., 123, 062206 (2005).

9 T. Kato and H. Kono, J. Chem. Phys., 128, 184102 (2008).

10 T. Yonehara and K. Takatsuka, J. Chem. Phys., 128, 154104 (2008).

${ }^{11}$ We apologize that the reference list in the above is far from complete.

12 K. Ando, J. Chem. Phys., 121, 7136-7143 (2004).

13 K. Ando, J. Chem. Phys., 125, 014104 (2006).

14 N. Sakumichi and K. Ando, J. Chem. Phys., 128, 164516 (2008).

15 A. A. Frost, in "Modern Theoretical Chemistry," ed. by H. F. Shaefer, Plenum, New York (1977) Vol. 3.

${ }^{16}$ L. Wilets, E. M. Henley, M. Kraft, and A. D. Mackellar, Nucl. Phys., A282, 341 (1977).
17 C. Dorso, S. Duarte, and J. Randrup, Phys. Lett. B, 188, 287 (1987).

18 D. Klakow, C. Toepffer, and P. G. Reinhard, J. Chem. Phys., 101, 10766 (1994).

19 A. Ono, H. Horiuchi, T. Maruyama, and A. Ohnishi, Prog. Theor. Phys., 87, 1185 (1992).

${ }^{20}$ H. Feldmeier and J. Schnack, Rev. Mod. Phys., 72, 655 (2000).

${ }^{21}$ J. T. Su and W. A. Goddard, Proc. Nat. Acad. Sci. USA, 106, 1001 (2009).

22 This form of the trial function in one-dimension has been studied in Refs 23 and 24, and called a 'semiquantal' ansatz in the latter. The semiquantal and semiclassical theories were distinguished as the classical nature emerges by restricting the Hilbert space in the former, while the classical trajectories are approximately quantized in the latter.

${ }^{23}$ F. Arickx, J. Broeckhove, E. Kesteloot, L. Lathouwers, and P. van Leuven, Chem. Phys. Lett., 128, 310 (1986).

24 A. K. Pattanayak and W. C. Schieve, Phys. Rev. E, 50, 3601 (1994).

25 P. Kramer and M. Saraceno, "Geometry of the Time-Dependent Variational Principle in Quantum Mechanics," Springer, Berlin (1981).

26 T. Marumori, T. Maskawa, F. Sakata, and A. Kuriyama, Prog. Theor. Phys., 64, 1294 (1980).

27 O. V. Prezhdo, Theor. Chem. Acc., 116, 206 (2006).

28 Y. Shigeta, H. Miyachi, T. Matsui, and K. Hirao, Bull. Chem. Soc. 
Japan, 81, 1230 (2008).

29 S. F. Boys, Proc. Roy. Soc., A200, 542 (1950).

${ }^{30}$ C. M. Reeves, J. Chem. Phys., 39, 1 (1963).

${ }^{31}$ L. Pauling and E. B. Wilson, "Introduction to Quantum Mechanics with Applications to Chemistry," Dover, New York (1985).

32 The Slater-type exponential functions are, however, not adequate for the floating basis as they introduce unnecessary cusps in the free space between the nuclei.

33 W. Heitler and F. London, Z. Phys., 44, 455 (1927).

${ }^{34}$ R. S. Mulliken, J. Chim. Phys., 46, 500, 521 (1949).

${ }^{35}$ Here we deal with a single VB configuration consisting of the perfect-pairing spin-couplings such as $\alpha \beta \cdot \alpha \beta$ and three others, and not with the resonance between the alternative spin-coupling configuration such as $\alpha \alpha \cdot \beta \beta$. Cases where the resonance is essential will be discussed elsewhere.

36 J. C. Slater, Phys. Rev., 38, 1109 (1931).

${ }^{37}$ M. H. Cohen and V. Heine, Phys. Rev., 122, 1821 (1961).

${ }^{38}$ N. W. Ashcroft, Phys. Lett., 23, 48 (1966).

39 W. C. Topp and J. J. Hopfield, Phys. Rev. C, 7, 1295 (1973).

${ }^{40}$ R. Brent, "Algorithms for Minimization without Derivatives," Dover, New York (2002).

${ }^{41}$ S. C. Wang, Phys. Rev., 31, 579 (1928).

${ }^{42}$ H. Shull and D. D. Ebbing, J. Chem. Phys., 28, 866 (1958).

43 A. C. Hurley, Proc. Roy. Soc. (London), A226, 179 (1954).

${ }^{44}$ H. Nakatsuji, S. Kanayama, S. Harada, and T. Yonezawa, J. Am. Chem. Soc., 100, 7528 (1978).

45 H. Huber, J. Mol. Struct. THEOCHEM, 76, 277 (1981).

46 T. Helgaker and J. Almlof, J. Chem. Phys., 89, 4889 (1988).
${ }^{47}$ K. Hirao and K. Mogi, J. Comp. Chem., 13, 457 (1992).

48 M. Tachikawa, K. Taneda, and K. Mori, Int. J. Quant. Chem., 75, 497 (1999).

49 W. Kolos and L. Wolniewicz, J. Chem. Phys., 49, 404 (1968).

${ }^{50}$ F. H. Crawford and T. Jorgensen, Phys. Rev., 47, 932 (1935).

51 W. Meyer and P. Rosmus, J. Chem. Phys., 63, 2356 (1975).

${ }^{52}$ K. Hirao, Chem. Phys. Lett., 190, 374 (1992).

${ }^{53}$ M. W. Schmidt, K. K. Baldridge, J. A. Boatz, S. T. Elbert, M. S. Gordon, J. H. Jensen, S. Koseki, N. Matsunaga, K. A. Nguyen, S. J. Su, T. L. Windus, M. Dupuis, and J. A. Montgomery, J. Comp. Chem., 14, 1347 (1993).

54 B. Ford, G. G. Hall, and J. C. Packer, Int. J. Quant. Chem., 4, 533 (1970).

55 To quote from Ref. 54, “... a single Gaussian is a such poor representation of the cusp, (...), that an outer function will try to move on to the nucleus to improve the representation there rather than to remain outside and represent the more weakly bound electrons".

56 R. J. Gillespie and E. A. Robinson, Angew. Chem. Int. Ed. Engl., 35, 495 (1996).

57 H. Nakai, Int. J. Quant. Chem., 107, 2849 (2007).

58 T. Ishimoto, M. Tachikawa, and U. Nagashima, J. Chem. Phys., 128, 164118 (2008).

59 A. Chakraborty and S. Hammes-Schiffer, J. Chem. Phys., 129, 204101 (2008).

60 A. Szabo and N. S. Ostlund, "Modern Quantum Chemistry," Dover, New York (1996). 